\title{
Clinical, epidemiological and therapeutic profile of patients with brachioradial pruritus in a reference service in dermatology*
}

\author{
Ana Cecília Versiani Duarte Pinto ${ }^{1}$ \\ Paula Yoshiko Masuda ${ }^{1}$
}

\author{
Patrick Alexander Wachholz ${ }^{2}$ \\ Antonio Carlos Ceribelli Martelli ${ }^{1}$
}

DOI: http:/ / dx.doi.org/10.1590/abd1806-4841.201644767

\begin{abstract}
This is a cross-sectional study, conducted from May to November $/ 2014$, in a dermatology reference unit, through review of medical records and interviews. In a sample of 49 patients with brachioradial pruritus, we observed higher prevalence of Caucasian $(81.6 \%)$ and women $(73.5 \%$ ), with a mean age of 56.1 years. Pruritus occurred in the topography of brachioradialis muscle in $87.8 \%$ of cases; $59.2 \%$ of the sample reported worsening of pruritus with sun exposure; the mean intensity of this symptom before treatment was 8.63 . Therapy effectiveness was described as "very good/good" in $79.2 \%$ of cases, and for $55.3 \%$ relapses were categorized as "uncommon".
\end{abstract}

Keywords: Case studies; Epidemiology, descriptive; Pruritus; Skin diseases; Skin manifestations

The brachioradial pruritus (BRP) is an uncommon neurodermatitis, of uncertain etiology. ${ }^{1,2,3}$ This condition is polymorphic, with itching, burning, stinging, or tingling sensations, particularly in the topography of the brachioradialis muscle (BRm), unilaterally or bilaterally. ${ }^{4,5}$ Occasionally, it extends to the upper arms, shoulders, back, and cervical region or upper thorax. ${ }^{4}$ The insidious clinical course, late diagnosis and poor or absent response to antihistamines and corticosteroids are associated with a significant impairment in the quality of life. ${ }^{1,4}$ Most publications involving BRP are case reports or case series ${ }^{4,5,6}$ and there is no standardization in the assessment of disease severity, impact and activity that allows direct comparison between studies. More comprehensive descriptions of BRP clinical presentation and response to treatment, with respect to the intensity of symptoms and therapeutic response, are gaps in the medical literature.

This study describes the clinical and therapeutic characteristics of patients with BRP followed in a dermatology reference unit, using reproducible patterns for measuring the intensity of symptoms and evaluation of therapeutic response.
This is a descriptive, cross-sectional, study, conducted between May and November 2014 through review of medical records and personal interviews. BRP diagnosis was defined as complaints lasting more than six weeks, according to a protocol for clinical and laboratory research of chronic pruritus, with a final sample of 49 patients. ${ }^{2}$

Data were analyzed in the software Sphinx Lexica 5.0 and SPSS 18.0, using descriptive statistics. This was a convenience sample, and the study was approved by the Ethics Review Board (CAAE-17133213.9.0000.5475).

BRP was more frequent in women $(73.5 \%)$ and Caucasian $(81.6 \%)$, with mean age of $56.1 \pm 10$ years. Time between onset of the pruritus and the first attendance in the unit ranged from 2 to 36 months, with mode of 24 months.

The intensity of pruritus was measured using a Visual Analogue Scale (0-10), with a mean of 8.63 before treatment. The onset of symptoms in BRm topography occurred in $87.8 \%$ of patients, bilateral involvement in $77.6 \%$, and intermittent symptoms in $73.5 \%$.

Received on 28.05.2015

Approved by the Advisory Board and accepted for publication on 25.08.2015

* Study performed at Instituto Lauro de Souza Lima(ILSL) - Bauru (SP), Brazil. Financial Support: None.

Conflict of Interest: None.

Instituto Lauro de Souza Lima (ILSL) - Bauru (SP), Brazil.

Universidade Estadual Paulista "Júlio de Mesquita Filho" (Unesp) - Botucatu (SP), Brazil.

C2016 by Anais Brasileiros de Dermatologia 
TABLE 1: Description of the main therapeutic options for the treatment of brachioradial pruritus, according to the amount of drugs used - Bauru (SP), Brazil, 2014

\begin{tabular}{lll}
\hline & Frequency $(\mathrm{n})$ & Percentage $(\%)$ \\
\hline None & 4 & 8.2 \\
Monotherapy & 29 & 59.2 \\
Combination of 2 drugs & 15 & 30.6 \\
Combination of 3 & 1 & 2.0 \\
or more drugs & & 100.0 \\
TOTAL & 49 & \\
\hline
\end{tabular}

The follow-up time ranged from 8 to 60 months, with a median of 35 months. In this period, recurrences were considered "uncommon" by $55.3 \%$ of patients, "frequent" by $29.8 \%$ and "very common" by $2.1 \%$. Among the patients, $12.8 \%$ remained without pruritus during follow-up.

BRP is a peculiar medical condition diagnosed with increasing frequency in Dermatology. Most studies described a higher prevalence of the disease in women, ${ }^{4,8}$ with mean ages greater than 50 years. ${ }^{3,4,6}$

TABLE 2: Description of drugs used in the treatment of brachioradial pruritus by pharmacologic class, frequency and percentage of citations - Bauru (SP), Brazil, 2014

\begin{tabular}{lll}
\hline & Citation frequency $(\mathrm{n})$ & Percentage $(\%)$ \\
\hline Tricyclic antidepressants & 22 & 33.3 \\
Benzodiazepine derivatives tricyclic antidepressants & 15 & 22.8 \\
Antipsychotics & 12 & 18.1 \\
Anticonvulsants & 07 & 10.6 \\
Serotonin reuptake inhibitor antidepressant & 04 & 6.1 \\
Antihistamines & 02 & 3.0 \\
None & 04 & 6.1 \\
TOTAL & 66 & 100.0 \\
\end{tabular}

We analyzed worsening factors for pruritus: for $51 \%$ of the sample it was worsened with touch; for $59.2 \%$, with sun exposure; for $75.5 \%$, with heat. For $91.8 \%$, the pruritus worsened with the act of scratching; the worsening of symptoms at night was confirmed by $69.4 \%$ of the sample.

The Ice-Pack signal was positive in $46.9 \%$ patients, of whom $43.5 \%$ reported "good reduction" of itching with local ice application. ${ }^{7}$

Anatomical changes in cervical thoracic spine were investigated by X-ray: reduction of the intervertebral spaces was found in $61.2 \%$, and C6-C7 topography was the most affected (69.4\%).

Monotherapy was used in $59.2 \%$ of cases. Traditional tricyclic antidepressants (TCAs; amitriptyline, for example) were used in $33.3 \%$ of patients; doxepin (dibenzoxepin derivative TCA) in $22.8 \%$, followed by antipsychotics $(18.1 \%)$ and anticonvulsants $(10.2 \%)$. The effectiveness of treatment was considered "very good" and "good" in $79.2 \%$ of cases; $12.5 \%$ of the patients did not observe changes in symptoms (Tables 1 and 2).
Considering that BRP can be a harrowing morbid condition, ${ }^{1}$ clinical characterization is necessary for the proper management. Worsening factors, such as touch, sun exposure, the act of scratching and seasonality - have been reported in other case series. ${ }^{2,4,5,6}$

Mean follow-up in this study was higher than that described in previous series. ${ }^{5,6}$ TCAs, antipsychotics and anticonvulsants are central modulators of pruritus. ${ }^{9}$ This study suggests that these drugs may be effective in BRP, reducing symptoms, as well as recurrences.

This survey has limitations related to the study design, the sample size and selection method. New studies should assess the validity and psychometric properties of measures of disease activity or response to treatment in BRP.] 


\section{REFERENCES}

1. Ständer S, Weisshaar E, Mettang T, Szepietowski JC, Carstens E, Ikoma A, et al. Clinical classification of itch: a position paper of the International Forum for the Study of Itch. Acta Derm Venereol. 2007:87:291-4.

2. Cassano N, Tessari G, Vena GA, Girolomoni G. Chronic pruritus in the absence of specific skin disease: an update on pathophysiology, diagnosis, and therapy. Am J Clin Dermatol. 2010;11:399-411.

3. Barry R, Rogers S. Brachioradial pruritus - an enigmatic entity. Clin Exp Dermatol. 2004;29:637-8.

4. Masuda PY, Martelli AC, Wachholz PA, Akumatsu HT, Martins AL, Silva NM. Brachioradial pruritus - descriptive analysis of Brazilian case series. J Dtsch Dermatol Ges. 2013;11:530-5.

5. Mirzoyev SA, Davis MDP. Brachioradial pruritus: Mayo Clinic experience over the past decade. Br J Dermatol. 2013;169:1007-15.

6. Veien NK, Laurberg G. Brachioradial pruritus: a follow-up of 76 patients. Acta Derm Venereol. 2011;91:183-5.

7. Bernhard JD, Bordeaux JS. Medical pearl: the ice-pack sign in brachioradial pruritus. J Am Acad Dermatol. 2005;52:1073.

8. Marziniak M, Phan NQ, Raap U, Siepmann D, Schürmeyer-Horst F, Pogatzki-Zahn $\mathrm{E}$, et al. Brachioradial pruritus as a result of cervical spine pathology: the results of a magnetic resonance tomography study. J Am Acad Dermatol. 2011;65:756-62.

9. $\quad$ Ständer S, Weisshaar E, Luger TA. Neurophysiological and neurochemical basis of modern pruritus treatmentExp Dermatol. 2008;17:161-9.

MAILING ADDRESS:

Patrick Alexander Wachholz

Rodovia Comandante João Ribeiro de Barros, km 225/226

Bauru

17034-971 - São Paulo - SP

Brazil

Email:p_wachholz@hotmail.com

How to cite this article:Pinto ACVD, Wachholz PA, Masuda PY, Martelli ACC. Clinical, epidemiological and therapeutic profile of patients with brachioradial pruritus in a reference service in dermatology. An Bras Dermatol. 2016:91(4):549-51. 